\title{
Internacionalización de la educación superior: redefiniciones, justificativas y estrategias
}

\section{Internationalization of higher education: redefinations, justifications and strategies}

\author{
José Camilo dos Santos Filho*
}

\section{Resumen}

El artículo tiene el objetivo de traer al debate la internacionalización de la educación superior a partir de tres aspectos: (1) Conceptos de internacionalización en distintas perspectivas; (2) Razones de la internacionalización; y (3) Estrategias de internacionalización, traducidas en un conjunto de actividades que operacionalizan la política de la institución universitaria. Se trata de investigación cualitativa, de cuño teórico. De entre los resultados, encontró que las primeras universidades europeas surgieron en el siglo XII ya internacionalizadas en su claustro docente y discente. En la era moderna, pocas universidades, más frecuentemente por los lazos coloniales, pasaron a recibir a estudiantes de sus colonias y/o de países menos desarrollados. Recientemente, las universidades pasaron a valorar la dimensión de la internacionalización. El concepto ha sido ampliamente discutido, pero aún no se ha llegado a un consenso sobre los indicadores más pertinentes para definir el grado de internacionalización de una universidad.

Palabras-clave: Educación superior. Estrategias de internacionalización. Internacionalización.

\section{Abstract}

The article aims to bring to the debate the internationalization of higher education from three aspects: (1) Concepts of internationalization in different perspectives; (2) Reasons for internationalization; And (3) Internationalization strategies, translated into a set of activities that operationalize the politics of the university institution. This is a qualitative, theoretical research. Among the results, it was found that the first European universities appeared in the twelfth century already internationalized in faculty and students. In the modern era, few universities, most often by colonial ties, began to receive students from their colonies and / or from less developed countries. Recently, universities have come to value the dimension of internationalization. The concept has been widely discussed, but a consensus has not yet been reached on the most relevant indicators to define the degree of internationalization of a university.

Keywords: Higher education. Internationalization strategies. Internationalization.

Recebido em 16/06/2017 - Aprovado em 19/11/2017

http://dx.doi.org/10.5335/rep.v25i1.8038

Doctor en Educación (Ph.D. in Education) por la University of Southern California (1971-1974). Realizó una etapa postdoctoral en la University of California at Los Angeles (Ucla). Actualmente es investigador colaborador en la Facultad de Educación de la Universidad Estadual de Campinas (Unicamp), actuando como coordinador del Grupo de Estudios e Investigaciones en Educación Superior. E-mail: jcamilosantos@gmail.com 


\section{Introducción}

Las primeras universidades europeas surgieron en el siglo XII ya internacionalizadas tanto en su claustro docente, como en el discente. Su ambiente cosmopolita fue dominante hasta el surgimiento de los estados nacionales modernos que pasaron a crear sus universidades nacionales. En la era moderna, tan sólo algunas pocas universidades de los países más avanzados más frecuentemente a causa de los lazos coloniales, pasaron a recibir a estudiantes de sus colonias y/o de países menos desarrollados científicamente, como fue el caso de países como China, Japón y Estados Unidos, que en la segunda mitad del siglo XIX enviaron a miles de jóvenes a estudiar en Europa, especialmente en Francia, Inglaterra y Alemania. En gran medida, fue esa generación de estudiantes internacionales que al volver han revolucionado las universidades de sus países de origen.

Más recientemente, en un movimiento más ampliado, las universidades de un considerable número de países del mundo pasaron a valorar la dimensión de la internacionalización (KERR, 1990; ALTBACH; KNIGHT, 2007) y a optar por ella para conocer nuevas experiencias y aprendizajes y generar conocimiento (KERR, 1990; MURPHY, 2007). Ante ese nuevo momento histórico vivido por las universidades nacionales, el concepto de internacionalización de la educación superior ha sido ampliamente discutido, pero aún no se ha llegado a un consenso sobre los indicadores más pertinentes para definir el grado de internacionalización de una universidad (DELGADO-MÁRQUEZ; HURTADO-TORRES; BONDAR, 2011).

En este texto traeremos al debate tres aspectos que consideramos relevantes para la comprensión del tema de la internacionalización de la educación superior: (1) Conceptos y redefiniciones de internacionalización de la educación superior; (2) Razones o rationales de la internacionalización de la educación superior; y (3) Estrategias de internacionalización de la educación superior.

\section{Conceptos de internacionalización de la educación superior}

Hay una variedad de términos relacionados con la palabra internacionalización de la educación superior, lo que ha creado alguna dificultad para un entendimiento consensual sobre el concepto o significado de ese término. Hans de Wit (2002) cita términos más genéricos, términos más específicos y concretos, y términos relacionados con el currículo. Entre los primeros enumera: educación internacional, estudios internacionales, internacionalismo, educación transnacional y globalización de la educación superior. Entre los segundos: movilidad académica, cooperación internacional, estudios en el exterior e intercambio internacional. En- 
tre los terceros: educación multicultural, educación intercultural, educación transcultural, educación para el entendimiento internacional, educación para la paz, educación global, estudios transnacionales, estudios globales.

Delgado-Márquez, Hurtado-Torres y Bondar (2011) elaboraron una clasificación de las definiciones de internacionalización de la educación superior propuestas por los especialistas en el tema, según cuatro perspectivas genéricas establecidas por Knight (1997): actividad, competición, ethos y proceso. Esas perspectivas están sintetizadas en el Cuadro 1.

Concebida en la perspectiva de la actividad, la internacionalización de la educación superior está definida por Harari (1992), Klasek (1992) y Mestenhauser y Ellingboe (1998) como el proceso de integración de la educación internacional en el currículo. Centrado en las actividades, este enfoque busca aumentar la cooperación internacional para garantizar a los países mayor seguridad nacional y mejor competitividad económica (POWELL, 2004).

Cuadro 1 - Perspectivas de internacionalización en el contexto de las instituciones de educación superior

\begin{tabular}{|c|c|}
\hline 1 Perspectiva de la actividad & Autores \\
\hline $\begin{array}{l}\text { Enfoca las actividades de educación superior que fomentan una dimensión } \\
\text { intercultural, incluida la presencia de los alumnos internacionales, } \\
\text { currículo e intercambio entre alumnos y profesores. }\end{array}$ & $\begin{array}{l}\text { Harari (1992) } \\
\text { Klasek (1992) } \\
\text { Arum y Van de Water (1992) } \\
\text { Mestenhauser y Ellingboe (1998) } \\
\text { Green y Olson (2003) } \\
\text { Javalgi, Griffith y White (2003) } \\
\text { Powell (2004) } \\
\text { Green y Shoenberg (2006) }\end{array}$ \\
\hline 2 Perspectiva de la competición & Autores \\
\hline $\begin{array}{l}\text { Señala el desarrollo de habilidades, de conocimientos y de valores } \\
\text { importantes para que se pueda competir en el mercado global. }\end{array}$ & $\begin{array}{l}\text { Soderqvist (2002) } \\
\text { Van der Wende (2007) } \\
\text { Ayoubi y Masoud (2007) } \\
\text { McGowan e Potter (2008) } \\
\text { Elkin, Farmsworth y Templer (2008) } \\
\text { Lipsett (2009) }\end{array}$ \\
\hline 3 Perspectiva del ethos & Autores \\
\hline $\begin{array}{l}\text { Subraya la creación de una cultura o entorno donde se valoran y se } \\
\text { apoyan las perspectivas y las iniciativas interculturales e internacionales. }\end{array}$ & $\begin{array}{l}\text { Pickert y Turlington (1992) } \\
\text { Hanson y Meyerson (1995) }\end{array}$ \\
\hline 4 Perspectiva del proceso & Autores \\
\hline $\begin{array}{l}\text { Enfatiza la integración de una dimensión internacional e intercultural } \\
\text { en la enseñanza, en la investigación y en los servicios a través de una } \\
\text { combinación de actividades, políticas y procedimientos. }\end{array}$ & $\begin{array}{l}\text { Knight (1994) } \\
\text { Schoorman (1999) } \\
\text { De Wit (2002) } \\
\text { Olson et al. (2001) }\end{array}$ \\
\hline
\end{tabular}

Fuente: Delgado-Márquez, Hurtado-Torres y Bondar (2011). 
En la perspectiva de la competición, la internacionalización de la educación superior está definida por Soderqvist como:

[...] o processo de transformação de uma instituição de educação superior nacional numa instituição de educação superior internacional, durante o qual se introduz uma dimensão internacional em todos os aspectos de sua gestão holística com o duplo objetivo de melhorar a qualidade do ensino e da aprendizagem e adquirir as competências desejadas (2002, p. 29).

Los beneficios esperados de la internacionalización de las universidades son la mejora de su calidad en relación con la enseñanza y la investigación y la posibilidad de autoevaluarse a la luz de los criterios internacionales (ELKIN; FARMSWORTH; TEMPLER, 2008; AYOUBI; MASOUD, 2007). El objetivo básico de la internacionalización de las universidades es captar a estudiantes extranjeros, estudiantes nacionales más cualificados e investigadores de alto nivel (VAN DER WENDE, 2007).

Desde el punto de vista del ethos, la internacionalización es un proceso de refuerzo del carácter internacional de una universidad mediante y apoyo de una institución líder (HANSON; MEYERSON, 1995; PICKERT; TURLINGTON, 1992).

Finalmente, desde la perspectiva del proceso, la internacionalización está definida por Jane Knight (1994) como un proceso de integración de la dimensión internacional/intercultural con la enseñanza, con la investigación y con los servicios de una universidad. En esa definición, la internacionalización tiene como característica un proceso dinámico que va hacia más allá de la simple realización de actividades en el ámbito internacional, considerándose precario el uso de ese criterio como indicador del grado de internacionalización de una institución de educación superior. Según Miura (2006), la constatación del crecimiento de la movilidad académica de estudiantes, profesores e investigadores, el incremento del número de cursos, programas y calificaciones con énfasis en temas internacionales y comparativos y el énfasis en el desarrollo de competencias internacionales e interculturales, entre otros elementos, han llevado a ese cambio conceptual. Concebida como un proceso de cambios internacionales provenidos desde la realidad global, la internacionalización tiene como indicadores, entre otros, la movilidad estudiantil, el desarrollo de investigaciones internacionales, la cooperación internacional, la existencia y actuación de asociaciones internacionales de educación superior, el enfoque de algunos sectores de las instituciones universitarias en la internacionalización, y la incorporación de la dimensión internacional en el currículo (BARTELL, 2003). 
La diversidad de definiciones de internacionalización de la educación superior ha llevado que De Wit (2002) alertara sobre el cuidado que se debe tener con el uso de ese término y a sugerir algunos parámetros básicos del concepto:

[...] à medida em que a dimensão internacional da educação superior ganha mais atenção e reconhecimento, as pessoas tendem a usar o tema da forma que melhor satisfaça aos seus propósitos. Uma definição mais focada é necessária para ser entendida com a importância que ela merece. Mesmo se não houver concordância sobre a definição, a internacionalização precisa ter parâmetros para ser avaliada e, portanto, contribuir com a educação superior. Este é o motivo pelo qual o uso de uma definição em construção com uma estrutura conceitual é relevante para a internacionalização da educação superior (DE WIT, 2002, p. 114).

Debido a los desafíos y cambios ocurridos en el mundo con el proceso de globalización e internacionalización de las universidades y de las sugerencias de De Wit (2002), Knight (2003) redefine su concepto de internacionalización formulado en 1994 y propone un nuevo concepto enfocando el proceso y los cambios. Para la autora, la internacionalización debe ser comprendida en los niveles nacional, sectorial e institucional. Por tanto, se hace necesaria una nueva definición que comprenda esos niveles y refleje las realidades actuales. De otra parte, esa definición no debe especificar las razones, beneficios, resultados, actores, actividades o proveedores de la internacionalización, pues esos elementos cambian de nación para nación y de institución para institución. Lo relevante es que la dimensión internacional se refiera a todos los aspectos de la educación y al papel por ella ejercido en la sociedad.

Tienendo en cuenta todas esas premisas, Knight propone la siguiente definición: "Internacionalização nos níveis nacional, setorial e institucional é definida como o processo de integração de uma dimensão internacional, intercultural, ou global no propósito, nas funções ou na oferta de educação pós-secundária" (2003, p. 2). En esa definición, el autor señala y explicita el sentido de algunos conceptos-chave. Entre ellos, cita los conceptos de proceso, de dimensión internacional, intercultural o global, de integración de esas dimensiones en las políticas, en los programas curriculares y en los procedimientos y, por último, los conceptos de propósito, funciones y oferta que se deben considerar en conjunto.

La internacionalización es un proceso dinámico, es decir, un esfuerzo continuado de cambio o evolución y no un conjunto de actividades aisladas. Ella comprende tres dimensiones: la internacional, la intercultural y la global, es decir, las relaciones entre naciones, culturas o países. En la dimensión intercultural, la internacionalización también se refiere a la diversidad étnica/cultural dentro de los países, de las comunidades e instituciones. La dimensión global, si bien controvertida y contaminada de críticas, es añadida para expresar el sentido de alcance mundial. Como enfatiza Knight (2003), hay una complementariedad entre esos tres términos que juntos expresan la riqueza de la amplitud y profundidad de la internacionalización. Como observan algunos teóricos de la internacionalización, la globalización económica ha impulsado la internacionalización de la educación 
superior y la internacionalización de la educación superior viene impulsando la globalización. Asimismo, Knight añade que "a internacionalização está transformando o mundo da educação e a globalização está transformando o mundo da internacionalização" (2003, p. 3).

El concepto de integración visa a denotar el proceso de incorporación de la dimensión internacional e intercultural en las políticas y programas para garantizar que esa dimensión permanezca central y sostenible y no tan sólo marginal. Knight (2003) propone que los conceptos de propósito, funciones y oferta sean entendidos de forma articulada y aclara su significado:

O propósito se refere ao papel global e aos objetivos da educação superior para um país ou à missão de uma instituição. A função se refere aos elementos ou tarefas principais que caracterizam um sistema pós-secundário nacional ou uma instituição individual. Geralmente estes incluem o ensino, a pesquisa e o serviço à sociedade. A oferta é um conceito mais restrito. Refere-se à oferta de cursos e programas educacionais seja domesticamente seja em outros países. Isto inclui a oferta por meio de instituições tradicionais de educação superior, mas também por novos provedores como empresas multinacionais que frequentemente estão mais interessadas na oferta global de seus programas e serviços do que na dimensão internacional ou intercultural de um campus ou das funções de pesquisa e serviço (KNIGHT, 2003, p. 3).

Cabe asimismo observar que Knight (2003), en su nueva definición, ha incluido también lo que denominó abordaje botton-up y top-down (de la base hacia la cumbre, de la cumbre hacia la base) para incluir la influencia importante del nivel nacional/sectorial en la dimensión internacional de la educación superior mediante la política, la financiación y los marcos reguladores.

Según Knight (2004), su nueva definición de internacionalización de la educación superior no contradice a la anterior, sino que la explicita y la refina con términos más genéricos, volviéndola generalizable a los diferentes contextos educacionales nacionales y no especificando las razones, beneficios, resultados, actores, actividades y patrocinadores de la internacionalización, que podrán ser peculiares en cada país. Posteriormente a esa definición Knight (2011, 2012a) en dos textos breves presentó cinco mitos y cinco verdades que explican y aclaran dimensiones relevantes de su definición de internacionalización de la educación superior. Los mitos de la internacionalización de la educación superior listados por Knight son:

Mito um. Estudantes estrangeiros como agentes de internacionalização: Mais estudantes estrangeiros no campus vão produzir uma cultura institucional e um plano de estudos mais internacionalizados; Mito dois. A reputação internacional como indicador de qualidade: Quanto mais internacional é uma universidade [...] melhor é sua reputação; Mito três. Acordos institucionais internacionais: Quanto maior é o número de acordos internacionais ou quanto mais associações a redes tenha uma universidade, mais prestigiosa e atrativa ela é; Mito quatro. Credenciamento internacional: Quanto mais estrelas de credenciamento internacional tem uma universidade, mais internacionalizada está e, por isso, melhor ela é; Mito cinco. Construção de marca global: Um plano de marketing internacional é o equivalente a um plano de internacionalização (2011, p. 14). 
Knight (2012a, p. 1) llamó a esos mitos de misconceptions, es decir, concepciones equivocadas sobre internacionalización. A esa lista de Knight, Hans de Wit, actual director del Center for International Higher Education, del Boston College, en los Estados Unidos, añadió nueve concepciones equivocadas o engañosas sobre la internacionalización de la educación superior. De su elenco tan sólo dos son semejantes a dos de los cinco mitos (1 y 3) descriptos por Knight. Las nueve pseudoconcepciones de internacionalización de la educación superior señaladas por De Wit (2011) son las siguientes:

1. La internacionalización es enseñanza en el idioma inglés;

2. La internacionalización es enseñanza de un curso internacional;

3. La internacionalización es igual que cumplir un programa de estudios con asuntos internacionales;

4. La internacionalización significa tener muchos estudiantes internacionales matriculados en la Institución;

5. La internacionalización se puede implantar con éxito con tan sólo unos pocos estudiantes internacionales en clase;

6. No hay necesidad de evaluar las competencias interculturales e internacionales necesariamente como tales;

7. Cuantos más acuerdos tiene, más internacionalizada es la institución;

8. La educación superior es internacional por su propia naturaleza;

9. La internacionalización es un objetivo en sí mismo.

Reconociendo la centralidad de la internacionalización de la educación superior en el mundo actual y la necesidad de claridad sobre lo que ella efectivamente significa, y motivada por la publicación de su artículo sobre los mitos o concepciones equivocadas sobre internacionalización, Knight (2012a) propuso cinco verdades o principios que podrán contribuir para aclarar e iluminar el concepto y la práctica de internacionalización en las instituciones de educación superior. Esas verdades pueden ser formuladas en los siguientes términos (KNIGHT, 2012a):

1. La internacionalización debe respetar y complementar la dimensión local;

2. La internacionalización es un proceso adaptable al perfil de cada institución o país;

3. En el proceso de internacionalización hay beneficios, riesgos y consecuencias no intencionadas;

4. La internacionalización no es un fin en sí misma;

5. Globalización e internacionalización son diferentes, pero asociadas.

Recientemente, como producto adicional de estudio para el Parlamento Europeo para identificar tendencias clave en las estrategias nacionales corrientes de in- 
ternacionalización en Europa y en el resto del mundo y para delinear un escenario para el futuro de la internacionalización de la educación superior en Europa, De Wit y Hunter (2015) han propuesto una revisión de la definición de internacionalización de Jane Knight comúnmente aceptada entre los especialistas del campo. La definición propuesta resultó formulada así: Internacionalización es:

[...] o processo intencional de integração de uma dimensão internacional, intercultural ou global no propósito, nas funções e na oferta de educação pós-secundária, a fim de aprimorar a qualidade da educação e da pesquisa para todos os estudantes e corpo docente e fornecer uma contribuição significativa para a sociedade (DE WIT; HUNTER, 2015, p. 3, destacados del autor).

La internacionalización de la educación superior como un proceso intencional se está volviendo la política dominante en los niveles nacional e institucional en muchos países del mundo y, en especial, en Europa. Sin embargo, mismo en Europa, vista como un caso de mejor práctica de internacionalización, De Wit y Hunter (2015) reconocen que todavía hay mucho que hacer y hay un grado variado de éxito y serios desafíos a superar en el sur, centro y este de Europa. Otro elemento enfatizado en la definición de internacionalización de la educación superior por De Wit y Hunter es que ella no es un fin en sí misma, sino un medio para perfeccionar la calidad de la educación y de la investigación, no debiendo basarse tan sólo en la justificativa económica. Finalmente, cabe señalar que esa definición refleja la consciencia creciente de que la internacionalización:

[...] deve tornar-se mais inclusiva e menos elitista não focando predominantemente a mobilidade, e sim mais o currículo e os resultados da aprendizagem. O componente "exterior" (mobilidade) precisa tornar-se uma parte integral do currículo internacional para assegurar a internacionalização para todos e não somente para a minoria móvel (DE WIT; HUNTER, 2015, p. 3).

La inclusión de la internacionalización "en casa" (at home) como un nuevo pilar en la estrategia de internacionalización abrirá un nuevo horizonte en las políticas de internacionalización de la educación superior tanto en nivel nacional como en el institucional para efectuarse concretamente.

Anteriormente a De Wit y Hunter (2015), Knight (2012b) ya había propuesto la conceptualización de la internacionalización como teniendo dos pilares íntimamente relacionados e interdependientes - el doméstico (at home, en casa) y el exterior (abroad, "más allá de la frontera", transfronterizo o transnacional). Por eso, Knight distingue dos tipos básicos de internacionalización: la internacionalización "en casa" o doméstica y la internacionalización en el exterior o "educación transfronteriza". Para la autora, la dimensión internacional del currículo, como una de las expresiones de la internacionalización en casa, está avanzando hacia la integración de las perspectivas internacional, global, intercultural y comparada en el proceso de enseñanza y aprendizaje y en los contenidos programáticos. La inclusión de conocimientos, habilidades y valores internacionales e interculturales 
constituye un nuevo énfasis curricular. La internacionalización en el exterior o transfronteriza tiene implicaciones significativas para la internacionalización basada en el campus de la universidad y viceversa. La movilidad estudiantil, como parte de la "educación más allá de la frontera", según Knight (2012b), es la gran carrera de cerebros (great brain race) del siglo XXI, con implicaciones para ganancia cerebral, evasión de cerebros y entrenamiento cerebral (brain gain, brain drain, brain train).

Basado en el Informe del $3^{\text {er }}$ Estudio Global de la Asociación Internacional de Universidades (AIU) publicado en 2010, Beelen (2011) presentó un análisis crítico de cuatro cuestiones de aquel estudio que tratan de la internacionalización en casa (IeC) y de la internacionalización del plan de estudios. El estudio del Informe de la AIU abarcó seis regiones del mundo (África, Asia y el Pacífico, Europa, América Latina y el Caribe, Oriente Medio y América del Norte), y tuvo como muestra a sujetos del $2^{\circ}$ y $3^{\circ}$ Estudio Global. En su análisis crítico, Beelen destacó las políticas o estrategias y las actividades de la internacionalización en casa, las bases o razones de la internacionalización del plan de estudios y los beneficios y las barreras a la internacionalización. Estudios más específicos sobre la internacionalización en casa ya habían sido realizados anteriormente por Beelen y colaboradores (2007) y Crowther et al. (2001), entre otros, lo que muestra la creciente valoración e implementación de ese nuevo pilar de la internacionalización de la educación superior con el propósito de volverla accesible a todos los estudiantes y no sólo a una minoría privilegiada en la versión "educación transfronteriza".

\section{Razones de la internacionalización de la educación superior}

Las razones o justificativas para la búsqueda de la internacionalización de la educación superior en los países y en las propias instituciones universitarias han sido múltiples y diferenciadas para países e instituciones. Knight y De Wit (1995) han clasificado esas razones en dos grandes categorías: (1) razones políticas y económicas y (2) razones culturales y educacionales. Otros autores reclasifican esas razones en cuatro categorías: (1) política; (2) económica; (3) sociocultural; y (4) académica.

Según De Wit (2002 apud Miura, 2006), esa reclasificación permite reorganizar más adecuadamente las principales razones que, de acuerdo con Knight (2005) impulsaron la internacionalización de la educación superior. Con la razón política están relacionadas la política externa, la seguridad nacional, la cooperación para el desarrollo, la paz y el entendimiento mutuo, la identidad nacional y regional. Ese tipo de justificativa alegan especialmente los gobernantes para adoptar una política nacional de internacionalización de la educación superior al reconocer que sus beneficios son superiores a sus costes. En general, esa razón política está 
considerada más importante en nivel nacional que en nivel internacional. A la razón económica se vinculan la competitividad, el crecimiento económico, el mercado laboral, la demanda nacional por educación y los incentivos financieros para las instituciones y gobiernos. Esa razón económica tiene importancia y relevancia crecientes en los países desarrollados que, para perfeccionar y mantener ventaja competitiva en la economía globalizada, buscan desarrollar una fuerza de trabajo altamente cualificada y competitiva, invertir en investigación aplicada y vender servicios educacionales para estudiantes de países extranjeros. Con la razón sociocultural se relacionan el desarrollo social y comunitario, el entendimiento intercultural y el vínculo con la identidad cultural. La actual economía globalizada y el sistema de información y comunicación han impactado y afectado la razón sociocultural. Con la razón académica se vinculan la promoción de la dimensión internacional de la investigación, de la enseñanza y de la extensión, el desarrollo de la institución, la reputación y el status internacional, la mejora de la calidad y los estándares académicos internacionales. Ella se relaciona directamente con el perfeccionamiento de la docencia, del proceso de aprendizaje y de la investigación. Según Knight (1997), esos cuatro tipos de razones no son totalmente distintos o exclusivos, pudiendo cambiar o evolucionar en respuesta a las necesidades y prioridades cambiantes de los países y de las instituciones.

Las razones para la adhesión a la internacionalización de la educación superior pueden cambiar tanto en nivel institucional como en nivel nacional. En el nivel institucional, las distintas circunstancias internas y externas a la propia institución y las particularidades y perfiles de cada universidad permiten una diversidad de interpretaciones de las razones para la internacionalización. En nivel nacional, la interferencia y actuación de organismos internacionales y el cambio de las políticas gubernamentales pueden interferir en las opciones gubernamentales por la implementación y soporte de determinadas formas de internacionalización. Como ha observado De Wit, cabe también registrar las siguientes constataciones:

1) Globalmente, há fortes razões dentro e entre diferentes grupos de provedores; Geralmente, os provedores não têm uma razão exclusiva para a internacionalização; 2) As razões podem diferir entre grupos de provedores e dentro de grupos de provedores; 3) As prioridades nas razões podem mudar ao longo do tempo e podem mudar por país e por região; 4) Em muitos casos, as razões têm motivos mais implícitos do que explícitos para a internacionalização (2002, p. 224).

Cinco han sido las razones para justificar la adhesión a la internacionalización de la educación superior en nivel institucional y cinco en nivel nacional (KNIGHT, 2004). Hemos adaptado y sintetizado esas razones de acuerdo con Mueller (2013) en el Cuadro 2. 
Cuadro 2 - Razones de la internacionalización en los niveles nacional e institucional

\begin{tabular}{|c|l|}
\hline Nivel & \multicolumn{1}{c|}{ Razones } \\
\hline \multirow{4}{*}{ Nacional } & Desarrollo de recursos humanos \\
& $\begin{array}{l}\text { Alianzas estratégicas } \\
\text { Transacciones comerciales } \\
\text { Construcción de la nación } \\
\text { Desarrollo social/cultural }\end{array}$ \\
\hline \multirow{4}{*}{ Institucional } & Reputación y perfil institucional \\
& Desarrollo de estudiantes y profesores \\
& Generación de receta \\
& Alianzas estratégicas \\
& Producción del conocimiento \\
\hline
\end{tabular}

Fuente: Mueller (2013), adaptado de Knight (2004, p. 23).

Para hacer más claras y explícitas las alegadas razones de la internacionalización de la educación superior tanto en nivel nacional como en nivel institucional, Knight (2004) presenta breve descripción de cómo las entienden los gobernantes o las instituciones de educación superior. En nivel nacional, el desarrollo de recursos humanos se dará por medio de estrategias, de reclutamiento e incentivo a la permanencia y a la formación de cuadro humano de alto nivel intelectual e intercultural capaz de contribuir para el desarrollo del país. Las alianzas estratégicas bilaterales o multilaterales podrán contribuir para la mejora de la enseñanza y el desarrollo de investigación conjunta, haciendo el sistema de educación superior más competitivo y generando beneficios culturales, políticos y económicos al país. Las transacciones comerciales en el ámbito de la educación superior han asumido distintas formas, tales como campus en el exterior, universidad en país extranjero, oferta de cursos para estudiantes extranjeros, encuadrando la educación superior entre los doce sectores de servicio, según la clasificación del General Agreement of Trade and Services (GATS) y concibiéndola como mercado y no como bien público. La construcción de la nación se basa en el desarrollo del país por medio de la cooperación técnica y de la mejora de la calidad de la enseñanza superior. El desarrollo cultural y social visa la promoción y comprensión de la identidad cultural nacional y de la interculturalidad.

En el nivel institucional, la reputación y perfil institucional se relacionan con los propósitos económico, político, social y académico. El renombre de la institución en el contexto regional y global podrá llevarla a cambiar las estrategias adoptadas y las razones de la internacionalización. Los rankings internacionales vienen afectando el reconocimiento de las instituciones y su crecimiento en respuesta la demanda internacional. El desarrollo de estudiantes y profesores en universidades extranjeras podrá contribuir para la formación adecuada de personal con las competencias demandadas en los niveles local y global. Universidades de algunos países están adhiriendo al proceso de internacionalización para generar receta, 
obteniendo beneficios financieros y complementando sus presupuestos. Alianzas estratégicas entre instituciones de educación superior se pueden caracterizar como nuevas formas de trabajo conjunto teniendo en cuenta beneficios mutuos en diferentes áreas como la tecnológica, científica, económica, social y académica.

Más recientemente, otras razones para internacionalizar una institución de educación son señaladas por Marmolejo y por Hénard, Diamond y Roseveare. Marmolejo (2010) identifica cinco razones, en orden de importancia:

- Perfeccionar la preparación del estudiante;

- Internacionalizar el currículo;

- Elevar el perfil internacional de la institución;

- Fortalecer la investigación y la producción de conocimiento;

- Diversificar su claustro docente y de funcionarios.

Hénard, Diamond y Roseveare (2012) indican otras seis razones para justificar la internacionalización de las instituciones de educación superior:

Aumentar la visibilidad nacional e internacional; Levantar la calidad institucional mediante asociaciones estratégicas; Agrandar la comunidad académica dentro de la que hace sus actividades volverse referencia; Movilizar los recursos intelectuales internos; Añadir importantes resultados de aprendizaje contemporáneo a la experiencia del estudiante; Desarrollar grupos de investigación más capacitados (2012, p. 9).

La internacionalización también se justifica en nivel nacional, porque ella habilita a los gobiernos a:

Desenvolver sistemas nacionais dentro de um quadro de referência global mais amplo; Produzir uma força de trabalho treinada com consciência global e competências multiculturais; Usar fundos da educação superior pública para promover a participação nacional na economia global do conhecimento; Beneficiar-se do comércio nos serviços da educação (HÉNARD; DIAMOND; ROSEVEARE, 2012, p. 9).

En el caso específico de Brasil, Lima y Contel (2009) investigaron las motivaciones y fases de la internacionalización de la educación superior en Brasil y constataron la presencia de cuatro períodos diferentes, identificando para cada periodo el programa, los proveedores y las motivaciones. La internacionalización de la educación superior brasileña se ha vinculado al proyecto de desarrollo del país, a las políticas de fortalecimiento de las instituciones de educación superior, a la creación, expansión y consolidación de los programas de posgrado stricto sensu, a la formación de profesores y al desarrollo de competencias necesarias al nuevo momento de la economía brasileña. El Cuadro 3, adaptado de Lima y Contel (2009) por Mueller (2013), sintetiza las informaciones básicas referentes a los programas, proveedores y motivaciones de los cuatro períodos de la internacionalización de la educación superior brasileña. 
Cuadro 3 - Periodos, programas, proveedores y motivaciones de la internacionalización de la educación superior brasileña

\begin{tabular}{|c|c|c|c|}
\hline Periodos & Programa & Proveedores & Motivación \\
\hline $\begin{array}{l}1{ }^{\text {er }} \\
\text { Periodo: } \\
\text { Años } 1930 \\
\text { y } 1950\end{array}$ & $\begin{array}{l}\text { - Programa de cooperación académica } \\
\text { internacional con énfasis en las misio- } \\
\text { nes que traían profesores }\end{array}$ & $\begin{array}{l}\text { - Universidades extran- } \\
\text { jeras y brasileñas }\end{array}$ & $\begin{array}{l}\text { - Académica: fortalecimiento } \\
\text { del proyecto académico de } \\
\text { las universidades emer- } \\
\text { gentes }\end{array}$ \\
\hline $\begin{array}{l}\text { 20 Periodo: } \\
\text { años } 1960 \\
\text { y } 1970\end{array}$ & $\begin{array}{l}\text { - Programa de cooperación académica } \\
\text { internacional con énfasis en la presen- } \\
\text { cia de consultores y en la concesión de } \\
\text { becas para realizar maestría/doctorado } \\
\text { en el exterior }\end{array}$ & $\begin{array}{l}\text { - Agencias internacio- } \\
\text { nales y gobierno bra- } \\
\text { sileño } \\
\text { - Agencias nacionales e } \\
\text { internacionales }\end{array}$ & $\begin{array}{l}\text { - Político-académica: rees- } \\
\text { tructuración del sistema de } \\
\text { educación superior en con- } \\
\text { sonancia con el "modelo } \\
\text { estadounidense" }\end{array}$ \\
\hline $\begin{array}{l}3^{\text {er }} \text { Periodo: } \\
\text { años } 1980 \\
\text { y } 1990\end{array}$ & $\begin{array}{l}\text { - Programa de cooperación académica } \\
\text { internacional con énfasis en la forma- } \\
\text { ción de grupos de estudio e investi- } \\
\text { gación alrededor de temas de interés } \\
\text { compartido; } \\
\text { - Concesión de becas para realizar doc- } \\
\text { torado en el exterior, en áreas clasifica- } \\
\text { das como estratégicas; } \\
\text { - Programa de cooperación académica } \\
\text { internacional con énfasis en la venida } \\
\text { de profesores visitantes, ida de estu- } \\
\text { diantes para realización de algunas } \\
\text { asignaturas }\end{array}$ & $\begin{array}{l}\text { - Agencias internacio- } \\
\text { nales y gobierno bra- } \\
\text { sileño } \\
\text { - Agencias nacionales e } \\
\text { internacionales } \\
\text { - Universidades extran- } \\
\text { jeras e instituciones } \\
\text { brasileñas de educa- } \\
\text { ción superior privadas }\end{array}$ & $\begin{array}{l}\text { - Académico-mercadológica: } \\
\text { a) Expansión y consolida- } \\
\text { ción de los programas } \\
\text { de posgrado stricto sen- } \\
\text { su; } \\
\text { b) Incremento de las inves- } \\
\text { tigaciones de punta en } \\
\text { áreas estratégicas; } \\
\text { c) Diferencial competitivo } \\
\text { de algunas instituciones } \\
\text { o de algunos cursos }\end{array}$ \\
\hline $\begin{array}{l}\text { 4 Periodo: } \\
\text { años } 2000\end{array}$ & $\begin{array}{l}\text { - Programa de cooperación académica } \\
\text { internacional con énfasis en la forma- } \\
\text { ción de grupos de estudio e investiga- } \\
\text { ción alrededor de temas estratégicos y } \\
\text { de interés partido; } \\
\text { - Concesión de becas para realizar doc- } \\
\text { torado en el exterior, en áreas clasifi- } \\
\text { cadas como estratégicas y sin tradición } \\
\text { de investigación en el país; } \\
\text { - Programa de cooperación académica } \\
\text { internacional con énfasis en la venida } \\
\text { de profesores visitantes, en la ida de } \\
\text { estudiantes para realización de algu- } \\
\text { nas asignaturas; } \\
\text { - Proyecto de creación de universidades } \\
\text { federales orientadas por la internacio- } \\
\text { nalización activa; } \\
\text { - Comercialización de servicios educa- } \\
\text { cionales. }\end{array}$ & $\begin{array}{l}\text { - Agencias internacio- } \\
\text { nales y gobierno bra- } \\
\text { sileño; } \\
\text { - Agencias nacionales e } \\
\text { internacionales; } \\
\text { - Universidades extran- } \\
\text { jeras e instituciones } \\
\text { brasileñas de educa- } \\
\text { ción superior priva- } \\
\text { das; } \\
\text { - Corporaciones inter- } \\
\text { nacionales; } \\
\text { - Universidades corpo- } \\
\text { rativas }\end{array}$ & $\begin{array}{l}\text { - Académica, política, econó- } \\
\text { mica y mercadológica; } \\
\text { a) Inserción internacional } \\
\text { de los programas de } \\
\text { posgrado stricto sensu; } \\
\text { b) Incremento de las inves- } \\
\text { tigaciones de punta en } \\
\text { áreas estratégicas; } \\
\text { c) Incremento regional de } \\
\text { carácter inclusivo; } \\
\text { d) Diferencial de algunas } \\
\text { instituciones o de algu- } \\
\text { nos cursos; } \\
\text { e) Captación de estudian- } \\
\text { tes }\end{array}$ \\
\hline
\end{tabular}

Fuente: Mueller (2013), adaptado de Lima y Contel (2009). 


\section{Estrategias de internacionalización de la educación superior}

Un conjunto de normas y directrices que presentan las prioridades de la institución caracterizan su política de internacionalización. La formulación de esa política en general está presente en la misión y en los valores de la institución de educación superior. Tradicionalmente, como observa Knight (2005), la internacionalización en nivel institucional se ha traducido en un conjunto de estrategias y actividades que operacionalizan su política institucional.

Estrategias son elementos del planeamiento institucional destinados a volver efectivo el proceso de internacionalización de la educación superior. Las estrategias y actividades constituyen la dimensión más operacional en el contexto de la internacionalización de la educación superior en nivel institucional. Ese término ganó relevancia cuando Knight (2004) y Knight y De Wit (1997) ampliaron el concepto de internacionalización de la educación superior y la definieron como un proceso que comprende estrategias programáticas y estrategias organizacionales que se deben integrar al plan de desarrollo institucional y a la cultura de la institución. En la elección y definición de esas estrategias, pondera Knight (2004), la universidad deberá vincularlas a las razones para la adopción de la política de internacionalización y a sus particularidades específicas.

Hans De Wit (2002 apud FARQUHAR, 2008) ha organizado las estrategias programáticas de internacionalización de la educación superior en cuatro categorías:

1) Programas acadêmicos - incluindo programas orientados para o estudante (tais como esquemas de mobilidade, trocas, trabalho e estudo no exterior), programas orientados para o corpo docente e de funcionários (tais como mobilidade docente e de funcionários, palestrantes visitantes e nomeações duplas para propósitos docentes) e atividades de desenvolvimento curricular (tais como internacionalização do currículo, estudo de língua estrangeira, estudos temáticos internacionais e de área, programas conjuntos e de duplo diploma); 2) Assistência técnica - incluindo construção de capacidade (tais como desenvolvimento de recurso humano, reforma institucional e inovação curricular), exportação de conhecimento focada para dentro (tais como recrutamento de estudantes internacionais por razões econômicas e desenvolvimento de cursos especiais baseados em lucro para eles e educação transnacional orientada para fora (tais como programas recepcionados por outras instituições, ofertas de educação a distância [crescentemente via internet], esquemas de articulação e integração, campus no exterior e arranjos de franquia; 3) Colaboração na pesquisa - incluindo programas orientados ao doutorado (tais como doutorados conjuntos e estudantes de doutorado internacionais), programas orientados para o corpo docente e de funcionários (incluindo mobilidade docente e de funcionários, especialistas visitantes e nomeações duplas para propósitos de pesquisa), e programas de desenvolvimento de pesquisa (incluindo projetos acadêmicos internacionais e acordos, bem como conferências internacionais e riscos de publicação, centros de tema de área e institutos de pesquisa conjugados; e 4) Atividades extracurriculares - incluindo clubes estudantis e associações, residências de docentes e funcionários e de estudantes internacionais, eventos interculturais, programas de ex-alunos internacionais, e projetos interculturais baseados na comunidade (2002 apud FARQUHAR, 2008, p. 1-2). 
Knight (2004) clasificó las estrategias de internacionalización de la educación superior en dos categorías - las estrategias programáticas y las estrategias organizacionales. Las estrategias programáticas abarcan cuatro subcategorías: programas académicos, investigación y colaboración científica, actividades nacionales e internacionales (domésticas y extranjeras) y actividades extracurriculares. Las estrategias organizacionales asimismo comprenden cuatro subcategorías: gobernanza, operaciones, servicios y recursos humanos. En el Cuadro 4 la autora presenta las actividades pertinentes a cada subcategoría de estrategia.

Cuadro 4 - Categorías de estrategias programáticas y organizacionales de internacionalización en nivel institucional

\begin{tabular}{|c|c|}
\hline \multicolumn{2}{|r|}{ Estrategias programáticas } \\
\hline Programas académicos & $\begin{array}{l}\text { - Intercambios de estudiantes } \\
\text { - Estudio de idiomas extranjeros } \\
\text { - Dimensión internacional del currículo } \\
\text { - Estudios temáticos } \\
\text { - Trabajo/estudio en el exterior } \\
\text { - Proceso de enseñanza y aprendizaje } \\
\text { - Programas de doble diploma } \\
\text { - Entrenamiento intercultural } \\
\text { - Movilidad de docentes/funcionarios } \\
\text { - Profesores y ponentes visitantes }\end{array}$ \\
\hline $\begin{array}{c}\text { Investigación y } \\
\text { colaboración científica }\end{array}$ & $\begin{array}{l}\text { - Área y centros temáticos } \\
\text { - Proyectos de investigación conjunta } \\
\text { - Conferencias y seminarios internacionales } \\
\text { - Artículos y trabajos publicados } \\
\text { - Acuerdos internacionales de investigación } \\
\text { - Programas de intercambio para investigación }\end{array}$ \\
\hline $\begin{array}{l}\text { Actividades nacionales } \\
\text { y transnacionales } \\
\text { (domésticas y } \\
\text { extranjeras) }\end{array}$ & $\begin{array}{l}\text { - Colaboración con grupos de organizaciones no-gubernamentales o grupos del } \\
\text { sector público-privado } \\
\text { - Servicio comunitario y proyecto de trabajo intercultural } \\
\text { - Vínculos, colaboraciones internacionales y redes } \\
\text { - Entrenamiento y programas de investigación } \\
\text { - Programa para ex-alumnos en el exterior }\end{array}$ \\
\hline \multicolumn{2}{|r|}{ Estrategias organizacionales } \\
\hline Gobernanza & $\begin{array}{l}\text { - Compromiso expreso por líderes } \\
\text { - Envolvimiento activo del claustro docente } \\
\text { - Razones y objetivos para la internacionalización bien articulados } \\
\text { - Reconocimiento de la dimensión internacional en la misión, planeamiento y do- } \\
\text { cumentos de política }\end{array}$ \\
\hline Operaciones & $\begin{array}{l}\text { - Vinculado al planeamiento, presupuesto y sistemas de revisión de cualidad en } \\
\text { nivel institucional y departamental } \\
\text { - Estructuras organizacionales apropiadas: sistemas formales e informales para } \\
\text { comunicación, relación y coordinación } \\
\text { - Equilibrio entre promoción centralizada y descentralizada y gestión de la interna- } \\
\text { cionalización } \\
\text { - Apoyo financiero adecuado y sistemas de destinación de recursos }\end{array}$ \\
\hline Servicios & $\begin{array}{l}\text { - Apoyo de unidades de servicios de la institución: alojamiento para estudiantes, } \\
\text { tecnología de la información } \\
\text { - Envolvimiento de unidades de apoyo académico: biblioteca, enseñanza y apren- } \\
\text { dizaje, desarrollo del currículo, entrenamiento de los docentes } \\
\text { - Servicios de apoyo estudiantil para estudiantes recibidos y enviados: Programas } \\
\text { de orientación, consejeros, entrenamiento cross-cultural, consejos sobre visados }\end{array}$ \\
\hline
\end{tabular}

Fuente: Knight (2004). 
Con vistas a construir un modelo de indicadores para abarcar el proceso de internacionalización en las instituciones de educación superior en sus más variadas formas de actividades, Brandenburg y Federkeil (2007) proponen la organización de tres grandes categorías principales de indicadores, es decir, los aspectos generales, la investigación académica y la enseñanza y estudio. La categoría "aspectos generales" comprende cinco subcategorías: gestión general, profesores, jóvenes investigadores, candidatos de doctorado y posdoctorado, técnico administrativo, recursos y red internacional. La categoría "investigación académica" abarca seis subcategorías: profesores, red internacional en investigación, recursos, proyecto de investigación internacional, resultados de investigación y joven investigador. La categoría "enseñanza y estudio" se explicita en ocho subcategorías: profesores, estudiantes de grado y de posgrado, servicio y administración, red internacional para enseñanza y aprendizaje, recursos, programas de estudio/currículo, graduados y reputación internacional. La lista de indicadores para cada categoría y respectivas subcategorías se presenta en el Cuadro 5.

Cuadro 5 - Modelo de indicadores de internacionalización

Continua

\begin{tabular}{|c|c|}
\hline \multicolumn{2}{|r|}{ Aspectos generales } \\
\hline Gestión en general & $\begin{array}{l}\text { - Relación del gestor con la internacionalización } \\
\text { - Acciones del gestor priorizan y/o incentivan la internacionalización }\end{array}$ \\
\hline Profesores & $\begin{array}{l}\text { - Experiencia y formación internacional de los profesores } \\
\text { - Reclutamiento de profesores internacionales } \\
\text { - Profesor visitante }\end{array}$ \\
\hline $\begin{array}{l}\text { Jóvenes investigadores, } \\
\text { candidatos de doctorado } \\
\text { y posdoctorado }\end{array}$ & $\begin{array}{l}\text { - Experiencia y formación en el exterior } \\
\text { - Candidatos del exterior }\end{array}$ \\
\hline $\begin{array}{l}\text { Técnico- } \\
\text { administrativo }\end{array}$ & $\begin{array}{l}\text { - Conocimiento de lengua extranjera } \\
\text { - Participación en programa de intercambio internacional } \\
\text { - Número de empleados en el exterior de Relaciones Internacionales o equivalente }\end{array}$ \\
\hline Recursos & $\begin{array}{l}\text { - Presupuesto para cooperación internacional con proporción del total de Institucio- } \\
\text { nes de Enseñanza Superior } \\
\text { - Número de funcionarios/plazas para atender Relaciones Internacionales }\end{array}$ \\
\hline Red internacional & $\begin{array}{l}\text { - Asociaciones activas } \\
\text { - Participación en eventos internacionales } \\
\text { - Miembro de redes internacionales }\end{array}$ \\
\hline \multicolumn{2}{|r|}{ Investigación académica } \\
\hline Profesores & $\begin{array}{l}\text { - Experiencia y formación internacional de los profesores } \\
\text { - Reclutamiento de profesores internacionales } \\
\text { - Profesor visitante }\end{array}$ \\
\hline $\begin{array}{l}\text { Red internacional en } \\
\text { investigación }\end{array}$ & $\begin{array}{l}\text { - Fondos externos } \\
\text { - Recursos derivados de proyectos con asociación internacional } \\
\text { - Número de comités y actividades, asociaciones profesionales internacionales } \\
\text { - Número de ediciones de periódicos internacionales } \\
\text { - Número de doctorados internacionales }\end{array}$ \\
\hline
\end{tabular}


Conclusão

\begin{tabular}{|c|c|}
\hline Recursos & $\begin{array}{l}\text { - Presupuesto de cooperación de investigación internacional y becas para doctorado } \\
\text { - Becas ofertadas por la Institución para candidatos internacionales al doctorado }\end{array}$ \\
\hline $\begin{array}{l}\text { Proyecto de } \\
\text { investigación } \\
\text { internacional }\end{array}$ & $\begin{array}{l}\text { - Número de proyectos con colaboración internacional } \\
\text { - Número de investigadores involucrados en proyecto de investigación con coope- } \\
\text { ración internacional } \\
\text { - Número de proyectos con fondos internacionales }\end{array}$ \\
\hline $\begin{array}{l}\text { Resultados de la } \\
\text { investigación }\end{array}$ & $\begin{array}{l}\text { - Número de publicaciones internacionales por investigador } \\
\text { - Número de citas por paper } \\
\text { - Número de contribuciones en conferencias internacionales por profesor } \\
\text { - Número de patentes internacionales por profesor }\end{array}$ \\
\hline Joven investigador & $\begin{array}{l}\text { - Experiencia y formación en el exterior } \\
\text { - Número de doble diplomas }\end{array}$ \\
\hline \multicolumn{2}{|r|}{ Enseñanza y estudio } \\
\hline Profesores & $\begin{array}{l}\text { - Número de profesores que imparten asignatura en lengua extranjera } \\
\text { - Número de profesores que pasan al menos un semestre en el exterior } \\
\text { - Actividad en el exterior como visitante } \\
\text { - Formación de doctorado en el exterior } \\
\text { - Experiencia profesional en el exterior } \\
\text { - Reclutamiento de otras nacionalidades }\end{array}$ \\
\hline $\begin{array}{l}\text { Estudiantes (grado y } \\
\text { posgrado) }\end{array}$ & $\begin{array}{l}\text { - Número de estudiantes internacionales } \\
\text { - Número de estudiantes de Instituciones de Enseñanza Superior en el exterior } \\
\text { - Número de estudiantes de doble diploma } \\
\text { - Práctica internacional }\end{array}$ \\
\hline Servicio y administración & $\begin{array}{l}\text { - Número de puestos para auxilio a estudiantes de intercambio } \\
\text { - Centro de orientación internacional } \\
\text { - Funcionarios internacionales } \\
\text { - Clases de aprendizaje intercultural } \\
\text { - Informaciones sobre países/culturas }\end{array}$ \\
\hline $\begin{array}{l}\text { Red internacional } \\
\text { para enseñanza y } \\
\text { aprendizaje }\end{array}$ & $\begin{array}{l}\text { - Número de estudiantes en intercambio in y out } \\
\text { - Número de estudiantes en in y out relativo al número de acuerdos } \\
\text { - Miembro activo de asociaciones especializadas en red internacional }\end{array}$ \\
\hline Recursos & $\begin{array}{l}\text { - Becas para periodo en el exterior } \\
\text { - Presupuesto para marketing en internacionalización, comparado al presupuesto } \\
\text { total } \\
\text { - Fondos para clases de visitantes } \\
\text { - Fondos y soporte para estancias propias en el exterior }\end{array}$ \\
\hline $\begin{array}{l}\text { Programas de Estudio/ } \\
\text { currículo }\end{array}$ & $\begin{array}{l}\text { - Proporción de cursos ofrecidos en lengua extranjera } \\
\text { - Currículos internacionales } \\
\text { - Número de lenguas extranjeras ofrecidas } \\
\text { - Reconocimiento de créditos en el exterior } \\
\text { - Ofertas de programas sobre interculturalidad }\end{array}$ \\
\hline Graduados & $\begin{array}{l}\text { - Egresados con doble diploma } \\
\text { - Egresados graduados de otra nacionalidad } \\
\text { - Informaciones sobre desarrollo profesional de los graduados }\end{array}$ \\
\hline Reputación internacional & $\begin{array}{l}\text { - Número de publicaciones internacionales por programa } \\
\text { - Número de estudiantes internacionales }\end{array}$ \\
\hline
\end{tabular}

Fuente: Mueller (2013), adaptado de Brandenburg y Federkeil (2007).

Para Brandeburg y Federkeil (2007), esa lista de indicadores podrá ayudar en la elaboración de estrategias institucionales, permitiendo posibles adaptaciones a la realidad de cada institución. Los autores recuerdan asimismo que diferentes or- 
ganismos nacionales o internacionales podrán proponer indicadores de internacionalización basados en distintas realidades y que, por ello, podrán ser inadecuados para la evaluación del proceso de internacionalización de determinado contexto nacional.

Entre los tres conocidos instrumentos de clasificación internacional de instituciones de educación superior - Times Higher Education Suplement (THES), Academic Ranking of World Universities (ARWU) utilizado por la Universidad Jiaotong de Shangai y Webometrics Ranking (WM usado por el Cybermetrics Lab) -, tan sólo el primero incluye la variable internacionalización entre sus criterios y trae dos indicadores relacionados con esa variable. El Cuadro 6 presenta una visión general de la metodología de la clasificación del THES.

Cuadro 6 - Visión general de la metodología de clasificación del THES

\begin{tabular}{|c|c|c|c|}
\hline Criterios & Indicador & Explicación & Peso \\
\hline \multirow{2}{*}{$\begin{array}{l}\text { Calidad de la } \\
\text { investigación }\end{array}$} & $\begin{array}{l}\text { Revisión por pares } \\
\text { académica global }\end{array}$ & $\begin{array}{l}\text { Puntuación compuesta a partir del cuestionario por } \\
\text { pares }\end{array}$ & $40 \%$ \\
\hline & Citas por docente & $\begin{array}{l}\text { Puntuación basada en la investigación en relación } \\
\text { con el tamaño del claustro de investigadores }\end{array}$ & $20 \%$ \\
\hline $\begin{array}{c}\text { Calidad de la } \\
\text { enseñanza }\end{array}$ & $\begin{array}{l}\text { Razón alumnos/ } \\
\text { profesores }\end{array}$ & Puntuación basada en la razón alumnos/profesores & $20 \%$ \\
\hline $\begin{array}{l}\text { Empleabilidad de los } \\
\text { graduados }\end{array}$ & $\begin{array}{l}\text { Revisión sobre el } \\
\text { empleo global }\end{array}$ & $\begin{array}{l}\text { Puntuación basada en las respuestas al } \\
\text { cuestionario de empleadores }\end{array}$ & $10 \%$ \\
\hline \multirow{2}{*}{ Internacionalización } & $\begin{array}{l}\text { Profesorado } \\
\text { internacional }\end{array}$ & $\begin{array}{l}\text { Puntuación basada en la proporción de profesorado } \\
\text { internacional }\end{array}$ & $5 \%$ \\
\hline & $\begin{array}{l}\text { Alumnado } \\
\text { internacional }\end{array}$ & $\begin{array}{l}\text { Puntuación basada en la proporción de alumnado } \\
\text { internacional }\end{array}$ & $5 \%$ \\
\hline
\end{tabular}

Fuente: Delgado-Márquez, Hurtado-Torres y Bondar (2011, p. 108).

\section{A modo de conclusión}

El proceso de internacionalización de la educación superior viene creciendo de manera acelerada, conllevando a su periódica reconceptualización para abarcar dimensiones inexploradas, poco valoradas o poco claras de esa nueva función de la universidad contemporánea. Al lado del crecimiento de la internacionalización por movilidad académica de estudiantes y docentes en consecuencia de las políticas nacionales fundadas en la expectativa de una economía del futuro basada en el conocimiento, resulta necesario implementar con más densidad la internacionalización con objeto de volver accesibles a todos los estudiantes los beneficios de la internacionalización en términos de conocimientos, habilidades y valores internacionales 
e interculturales necesarios a los jóvenes en el futuro para su efectiva integración al mercado laboral en una economía crecientemente globalizada.

De la internacionalización de la educación superior entendida e implementada como movilidad de personas como instrumento de cooperación de los países desarrollados en beneficio de los países en desarrollo mediante becas de los gobiernos anfitriones y de los propios Estados beneficiarios para un número reducido de privilegiados, se camina de modo acelerado hacia una internacionalización transfronteriza de distintas formas de movilidad académica y a una internacionalización "en casa" para viabilizar la inclusión ampliada de la dimensión internacional en la formación de la mayoría de los estudiantes que no tienen oportunidad de acceso a la movilidad académica transfronteriza.

La internacionalización de la educación superior como instrumento de cooperación de los países desarrollados a los países en desarrollo por razones académicas, culturales y políticas viene evolucionando para la internacionalización por razones económicas para oferta de servicios educacionales como "mercancía" cara a ser vendida a los países menos desarrollados. La consecuencia de ese movimiento ha sido la internacionalización como competición por mercados educacionales, la clasificación de la excelencia de las universidades como instrumento de marketing educacional, el capitalismo académico, el productivismo académico, la competición académica dentro de las propias universidades, la búsqueda de financiamiento privado para la investigación académica, el entendimiento de la educación superior como bien privado. El objetivo de la mejora de la excelencia académica de las universidades por la vía de la cooperación académica arriesga desvirtuarse en su batalla por fines más nobles.

Las estrategias programáticas y organizacionales de internacionalización de la educación superior, sea para la vía transfronteriza, sea para la vía doméstica, necesitan ser definidas mediante indicadores operacionales de su implementación para que puedan ser evaluadas periódicamente con base a criterios claros y definidos de esa política. La implementación efectiva de esa política implica en el comprometimiento efectivo, sea en nivel estatal, sea en nivel institucional, con la incorporación explícita de la dimensión de la internacionalización en los propósitos y funciones actuales de la universidad contemporánea. 


\section{Referencias}

ALTBACH, P. G.; KNIGHT, J. The internationalization of higher education: motivations and realities. Journal of Studies in International Education, SAGE Publications, v. 11, n. 3-4, p. 290-305, Fall/Winter 2007.

ARUM, S.; VAN DE WATER, J. The Need for a Definition of International Education in US Universities. In: KLASEK, C. (Ed.). Bridges to the future: strategies for internationalizing higher education. Carbondale: Association of International Education Administrators, 1992. p. 191-203.

AYOUBI, R.; MASSOUD, H. The strategy of internationalization in Universities. International Journal of Educational Management, v. 21, n. 4, p. 339-349, 2007.

BARTELL, M. Internationalization of universities: a university culture-based framework. Higher Education, Winipeg (Manitoba), v. 45, n. 1, p. 43-70, 2003.

BEELEN, J. et al. (Ed.). Implementing internationalization at home. Amsterdam: EAIE, 2007.

BEELEN, J. La internacionalización en casa en una perspectiva global: un estudo crítico del Informe del 3er Estudio Global de la AIU. Revista de Universidad y Sociedad del Conocimiento, Barcelona, v. 8, n. 2, p. 85-100, 2011.

BRANDENBURG, U.; FEDERKEIL, G. How to measure internationality and internationalization of higher education institutions! Indicators and key figures. Berlin: Centre for higher education development, 2007. (Working Paper, n. 92).

CROWTHER, P. et al. Internationalization at home: a position paper. Amsterdam: EAIE, 2001.

DELGADO-MÁRQUEZ, B. L.; HURTADO-TORRES, N. E.; BONDAR, Y. La internacionalización en la enseñanza superior: investigación teórica y empírica sobre su influencia en las clasificaciones de las instituciones universtitarias. Revista de Universidad y Scociedad del Conocimiento, v. 8, n. 2, p. 101-120, 2011.

DE WIT, H. Internationalization of higher education in the United States of America and Europe: a historical comparative and conceptual analysis. Westport, CT: Greeenwood Press, 2002.

DE WIT, H. Internationalization of higher education: nine misconceptions. International Higher Education, Boston, n. 34, p. 6-7, Summer 2011.

DE WIT, H.; HUNTER, F. The future of internationalization of higher education. International Higher Education, Boston, n. 83, p. 23, 2015.

ELKIN, G.; FARMSWORTH, J.; TEMPLER, A. Strategy and the internacionalization of Universities. International Journal of Educational Management, v. 22, n. 3, p. 239-250, 2008.

FARQUHAR, R. H. European Universities and Their International Perspectives. 2008. p. 1-6. Disponível em: <https://eric.ed.gov/?id=ED504364>. Acesso em: 15 jul. 2016.

GREEN, Madeleine; OLSON, Christa. Internationalizing the campus: a strategic approach. International Educator, v. XII, n. 1, p. 13-21, 2003.

GREEN, Madeleine; SHOENBERG, R. Where faculty live: internationalizing the disciplines. Washington, DC: American Council on Education, 2006.

HANSON, K.; MEYERSON J. International challenges to American Colleges and Universities: looking ahead. Phoenix, AZ: American Council on Education and The Oryx Press, 1995. 
HARARI, M. The internationalization of the curriculum. In: KLASEK, C. (Ed.). Bridges to the future: strategies for internationalizing higher education. Carbondale, IL: Association of International Education Administrators, 1992. p. 52-79.

HÉNARD, F.; DIAMON, L.; ROSEVEARE, D. Approaches to internationalization and their implications for strategic management and institutional practice: a guide for higher education institutions. OECD Higher Education Programme IMHE, 2012. Disponível em: <https://www. oecd.org/edu/imhe/Approaches\%20to\%20internationalisation\%20-\%20final\%20-\%20web.pdf>. Acesso em: 15 jul. 2016.

JAVALGI, R. G.; GRIFFITH, D.A.; WHITE, D. S. An empirical examination of factors influencing the internationalization of service firms. Journal of Services Marketing, v. 17, n. 2, p. 185-201, 2013.

KERR, C. The internationalization of learning and the nationalization of the purposes of higher education: Two "laws of motion" in conflict? European Journal of Education, v. 25, n. 1, p. 5-22, 1990.

KLASEK, C. (Ed.). Bridges to the future: strategies for internationalizing higher education: Carbondale, IL: Association of International Education Administrators, 1992.

KNIGHT, J. Internationalization of higher education: a conceptual framework. In: KNIGHT, J.; DE WIT, H. (Org.). Internationalization of higher education in Asia Pacific countries. Amsterdam: European Association for International Education, 1997. p. 5-19.

KNIGHT, J. Updating the definition of internationalization. International Higher Education, Boston, n. 33, p. 2-3, Fall 2003.

KNIGHT, J. Internationalization remodeled: definition, approaches, and rationales. Journal of Studies in International Education, SAGE Publications, v. 8, n. 1, p. 5-32, Spring 2004.

KNIGHT, J. Un modelo de internacionalización: respuesta a nuevas realidades y retos. In: DE WIT, Hans; JARAMILLO, Isabel Cristina; GACEL-AVILA, Jocelyne; KNIGHT, Jane. Educación Superior en América Latina: la dimensión internacional. Bogotá: Banco Mundial en coedición con Mayol Ediciones S. A., 2005. p. 1-38.

KNIGHT, J. Five myths about internationalization. International Higher Education, Boston, n. 6, p. 14-15, Winter 2011.

KNIGHT, J. Five truths about internationalization. International Higher Education, Boston, n. 69 , p. 1-4, Fall 2012a.

KNIGHT, J. Student mobility and internationalization: trends and tribulations. Research in Comparative and International Education, v. 7, n. 1, p. 20-33, 2012b. Disponível em: <http:// dx.doi.org/10.2304/rcie.2012.7.1.20>. Acesso em: 17 jul. 2016.

KNIGHT, J.; DE WIT, H. (Ed.). Internationalization of higher education in Asia Pacific countries. Paris: Organization for Economic Cooperation \& Development, Case Studies and Stocktake; Canberra: DEETYA, 1997.

KNIGHT, J.; DE WIT, H. Strategies for internationalization of higher education: historical and conceptual perspectives. In: DE WIT, H. (Ed.). Strategies for internationalization of higher education. Amsterdam: EAIA, 1995. p. 5-32. 
LIMA, M. C.; CONTEL, F. B. Períodos e motivações da internacionalização da educação superior brasileira. In: COLÓQUIO DA IFBAE, 5., maio 2009, Genebra. Disponível em: <http://www. ifbae.com.br/congresso5/pdfB0095.pdf>. Acesso em: 14 jul. 2016.

LIPSETT, A. Rise in university gender pay gap. The Guardian, 30 March 2009. Disponível em: $<$ http://www.guardian.co.uk/education/2009/mar/30/rise-universities-gender-pay-gap>. Acesso em: 18 maio 2017.

MARMOLEJO, F. Internationalisation of higher education: the good, the bad and the unexpected. Chronicle of Higher Education, Washington, DC, October 22, 2010. Disponível em: <http:// www.chronicle.com/blogs/worldwise/internationalization-of-higher-education-the-good-the-badand-the-unexpected/27512>. Acesso em: 10 jul. 2016.

MCGOWAN, S.; POTTER, L. The implications of the Chinese learner for the internationalization of the curriculum: an Australian perspective. Critical Perspectives on Accounting, v. 19, p. 181-198, 2008.

MESTENHAUSER, J. A.; ELLINGBOE, B. J. Reforming the higher education curriculum: internationalizing the campus. Phoenix, AZ: American Council on education \& Oryx Press, 1998.

MIURA, I. K. O processo de internacionalização da Universidade de São Paulo: um estudo em três áreas de conhecimento. Tese (Livre Docência) - Faculdade de Economia, Administração e Contabilidade, Universidade de São Paulo, Ribeirão Preto, 2006.

MURPHY, M. Experiences in the internationalization of education. Strategies to promote equality of opportunity at Monterray Tech. Higher Education, v. 53, n. 2, p. 167-298, 2007.

MUELLER, C. V. O processo de internacionalização do ensino superior: um estudo de caso da Universidade Federal do Rio Grande do Sul. 2013. 178 f. Dissertação (Mestrado em Relações Internacionais) - Instituto de Filosofia e Ciências Humanas, Universidade Federal do Rio Grande do Sul, Porto Alegre, 2013.

OLSON, David M. et al. Terrestrial Ecoregions of the World: a new map of life on earth: a new global map of terrestrial ecoregions provides an innovative tool for conserving biodiversity. BioScience, v. 51, n. 11, p. 933-938, 2001.

PICKERT, S.; TURLINGTON, B. Internationalizing the undergraduate curriculum: a Handbook for Campus leaders. Washington, DC: American Council on Education, 1992.

POWELL, C. Statement for International Education Week. U.S. State Department. 2004. Disponível em: <http://state.gov.iew/statements/powell.htm>. Acesso em: 15 jul. 2016.

SCHOORMAN, David. Unpackaging the multiple aspects of time in polychronicity. Journal of Managerial Psychology, v. 14, n. 3/4, p. 323-345, 1999.

SODERQVIST, M. Internationalization and its management at higher education institutions: applying conceptual, content and discourse analysis. Helsinki: Helsinki School of Economics, 2002.

VAN DER WENDE, M. Internationalization of higher education in the OECD countries: challenges and opportunities for the coming decade. Journal of Studies in International Education, v. 11, n. 3-4, p. 274-289, Fall-Winter 2007. 